\section{Looking for a good story}

\author{
R. W. Guillery
}

Eye, Brain, and Vision. By David $\mathrm{H}$. Hubel. W.H. Freeman:1988. Pp.240. f14.95. *

Our ideas about the role that individual nerve cells may play in perceptual experience owe much to relatively few people. David Hubel and Torsten Wiesel contributed significantly to the development of these ideas. In the 1960s and 1970s, working first at Johns Hopkins and then at Harvard, they showed how cells in the visual cortex respond to simple but rigorously specified visual stimuli. They described the characteristics of these stimuli and classified cortical cells accordingly. They went on to show that the arrangement of cortical cells can be analysed in terms of cortical 'columns' and 'hypercolumns', and these came to be seen as basic units of cortical organization in general. In addition, and perhaps most interestingly, they demonstrated that the development of normal response properties in cortical cells depends upon normal visual experience early in life. Early deprivation in one eye, or both, produces severe and irreversible visual losses, which are due to an abnormal development of the pathways that pass from one eye to the cortex.

In Eye, Brain, and Vision David Hubel summarizes these studies, setting them in relation to early observations on the retina, the nerve impulse and synaptic transmission. He shows how his own work with Wiesel grew out of the now classical analyses of Hodgkin, Huxley, Eccles, Katz and Kuffler. It makes a good story and is clearly presented for the general reader, ". . . with a scientific training but not an expert in biology, let alone neurobiology".

As would be expected, the book owes much to the Scientific American approach. Problems are stated in as simple and straightforward a manner as possible, and solutions are discovered by people with two names and one place of work (here not always correctly given). The text is laced by Hubel's personal style, which favours jokes and occasionally uses humour to dispose of difficult issues. He avoids complex terms where simple ones can be found, and this is generally, but not always, helpful. 'Nucleus', which is awkward because it has two different meanings in neurobiology, causes trouble. First it appears as 'globs', then 'balls' but finally turns into 'nucleus'.

Most of the book deals with experi-

* In the United States published as volumes in the Scientific American Library. ments done in the 1960s and 1970s, and could well have been written in the mid1970 s. Not only the visual pathways, but the nerve cells they use belong to this era. Important recent research on multiple visual areas receives only brief mention, and neither parallel ' $X$ ', ' $Y$ ' and ' $W$ ' pathways nor studies demonstrating a role for inhibitory interneurons in the cortex have a part in the book. Discussion of some of Hubel and Livingstone's more recent work on colour-opponent cells in the 'blobs' of the visual cortex is included as a brief look into the 1980s.

There is an important and puzzling departure from general usage in the account of cortical 'modules'. Hubel's suggestion that the modules are larger in cortical layers 3 and 5 than in layer 4 goes entirely counter to current concepts of what a module is. The argument is interesting, deriving from the original defini-

tion of a hypercolumn, and I suspect that, if followed to its logical conclusion, the argument might lead to the (not unwelcome) death of the module.

The book is well illustrated with striking colour photographs and micrographs. It also has some unlikely drawings of nerve cells, which look more like old-fashioned kites flying in the cortex than like neurons. Because they purport to show how simple and complex cells are interconnected, the resemblance may be intended. Finally, there are some marvellously revealing glimpses of Ramón y Cajal playing chess and of the Harvard neurobiologists at play. They provide a clue to the mixture of serious and frivolous that has long given Hubel's style a most readable quality.

R.W. Guillery is the Professor in the Department of Human Anatomy, University of Oxford, South Parks Rpad, Oxford OXI $3 Q X$, $U K$.

\section{Picture chemistry}

\section{Murray Goodman}

Molecules. By P. W. Atkins. W.H. Freeman: 1987. Pp.197. £14.95.*

IN THE preface to this book, P. W. Atkins describes the beauty and joy he finds in molecular structures. He has selected topics for inclusion which appeal to him and which are evident in the world about us. There is no particular sequence to the chapters in which subjects are covered; rather, Dr Atkins takes a phenomenological view of molecules and their effects. Generally, the molecules are grouped into more or less integrated chapters in which very attractive photographs are coupled to brief accounts of molecular properties.

The book contains descriptions of molecules as simple as diatomic structures and as complex as macromolecules. Should the reader desire, it is possible to find information on structures which elicit taste and smell; which are involved in colour and vision, and smog and air pollution; and which act as drugs and even form materials. The facts are briefly described but the underlying science and interrelationships are omitted. In the introduction, Dr Atkins uses ball and stick representations, assigning colours to denote specific atoms that make up the molecules, and throughout the book the ball and stick structures, space-filling models and structural formulae are presented in a readily comprehensible fashion.

In "Simple Substances", the first chapter, the author describes small molecules that are claimed to be the basis for smog, pollution and acid rain; the relationships presented here are too elementary. In like manner, Chapter 2 contains a systematic treatment of hydrocarbon molecules and simple alcohols. Suddenly, there is a leap to gamma-aminobutanoic acid, a neurotransmitter. The brief explanations offered for the neurochemical interactions of ethanol, valium and gamma-aminobutanoic acid once again are oversimplified (incidentally, I don't see how one can tell the chirality of lactic acid from crystal birefringence, as is claimed in the caption to the photograph on p.49).

The chapter on synthetic and natural polymers is the best in the book because polymer structures are directly related to their properties. Dr Atkins is, therefore, able to tie the structure of various polymeric systems to their applications as fibres, engineering plastics, elastomers, adhesives and so on. In the last three chapters, a grasp of complex molecular reactions is necessary to understand the relevant effects. But the glossary-like presentations do not provide the necessary insight, and when the author outlines receptor sites such as on pp.44 (protein channels), 107 (taste), 125 (odour) and 165 (morphine), he uses cartoons and structures which are scientifically naive.

What, then, is the value of this book? Those seriously involved in chemistry will find it of little use. But for the chemically uninformed it is an eyecatching introduction to certain molecular effects, and students could be attracted by the photographs and magazine-like brevity of the text. It has often been said scientists do not communicate the content of their work to the public. Chemistry in particular suffers from a poor image. This book could serve the useful purpose of interesting people in the molecular world about us, and thus help to establish chemistry as the central science devoted to molecular studies.

Murray Goodman is a Professor in the Department of Chemistry, University of California, San Diego, La Jolla, California 92093, USA. 